\title{
O ensino da ecologia em diálogo com a cultura afro-brasileira na formação inicial de professores de ciências e biologia: uma análise da disciplina de metodologia de ensino em ecologia
}

The teaching of ecology in dialogue with afro-brazilian culture in the initial science and biology teachers training: an analysis of the discipline of teaching methodology in ecology

La enseñanza de la ecología en dialogo con la cultura afro-brasileña en la formación inicial de profesores de ciencias y biología: un análisis de la disciplina de metodología de enseñanza en ecología

Bruna Mayrinck de Freitas Rezende Graduada - Licenciatura em Ciências Biológicas, UFLA, Brasil brunachristina2011@yahoo.com.br

Isabela Alberico Sousa Graduada - Licenciatura em Ciências Biológicas, UFLA, Brasil ialberico@outlook.com

Ellen Gonzaga Lima Souza Professora Doutora, UFLA, Brasil ellenl.souza@ded.ufla.br

Antonio Fernandes Nascimento Junior Professor Doutor, UFLA, Brasil toni_nascimento@yahoo.com.br 


\begin{abstract}
RESUMO
A cultura afro-brasileira sempre foi muito desvalorizada no processo de formação do Brasil e do povo brasileiro. Foi nessa perspectiva que a disciplina de Metodologia do Ensino em Ecologia da Universidade Federal de Lavras trouxe a proposta de se trabalhar os conteúdos de Ecologia em diálogo com a cultura afro-brasileira. Essa visava que os licenciandos conhecessem, reconhecessem e valorizassem essa cultura. Dessa forma, o presente trabalho tem como objetivo investigar se os alunos conseguiram estabelecer um diálogo entre a cultura afro-brasileira e o ensino em ecologia e como o fizeram. Para essa análise foi utilizado o método qualitativo, e dentro deste a análise de conteúdo com a categorização. Com essa análise percebemos a importância dessa temática na formação de professores, para que esses possam levar para as salas de aula um ensino contextualizado e transversal, que reconheça a contribuição desse povo para essa sociedade plural brasileira.
\end{abstract}

PALAVRAS-CHAVE: Cultura Afro-brasileira. Formação inicial de professores. Ciências e Biologia.

\title{
ABSTRACT
}

Afro-Brazilian culture has always been greatly undervalued in the formation process of Brazil and the Brazilian people. From this perspective that the discipline of Ecology Teaching Methodology of the Federal University of Lavras brought the proposal of working the contents of Ecology in dialogue with the Afro-Brazilian culture. This aim was for the licenciandos to know, recognize and value this culture. Thus, the present work aims to investigate if the students succeeded in establishing a dialogue between Afro-Brazilian culture and teaching in ecology and how they did it. For this analysis was used the qualitative method, and within this the analysis of content with the categorization. With this analysis we perceive the importance of this theme in teacher training, so that they can take to the classroom a contextualized and transversal teaching that recognizes the contribution of these people to this Brazilian plural society.

KEYWORDS: Afro-Brazilian culture. Initial teacher training. Science and Biology.

\section{RESUMEN}

La cultura afro-brasileña siempre fue muy desvalorizada en el proceso de formación de Brasil y del pueblo brasileño. En esta perspectiva, la disciplina de Metodología de la Enseñanza en Ecología de la Universidad Federal de Lavras trajo la propuesta de trabajar los contenidos de Ecología en concordancia con la cultura afro-brasileña. Este pretendía que los futuros licenciados conocieran, reconocieran y valorasen esa cultura. De esta forma, el presente trabajo tiene como objetivo investigar cómo los alumnos de la disciplina mencionada anteriormente lograron establecer una concordancia entre la cultura afro-brasileña y la enseñanza en ecología. Para este análisis se utilizó el método cualitativo, y dentro de éste el análisis de contenido, proponiendo una categorización. Con ese análisis percibimos la importancia de esta temática en la formación de profesores, para que estos puedan llevar a la sala de aulas una enseñanza contextualizada y transversal, que reconozca la contribución de ese pueblo a esa sociedad pluricultural brasileña.

PALABRAS CLAVE: Cultura Afro-brasileña. Formación inicial de profesores. Ciencias y Biología. 


\section{INTRODUÇÃO}

Conhecido como um dos países que possuem uma maior miscigenação, o Brasil se destaca pela sua história e diversidade cultural de seu povo, influenciada por variadas etnias e culturas.

A formação histórica e cultural do Brasil é muito marcada pelas culturas indígenas e africana, vivenciando processos de encontros e desencontros entre esses povos e os invasores portugueses (NASCIMENTO 2013; ROCHA, 2013). Mas, segundo Rocha (2013), as contribuições dessas culturas para a formação do povo brasileiro não tiveram notoriedade ao longo da história. Justifica-se isto pelo fato de que a luta pela igualdade dos direitos dos afro-brasileiros não terminou com a abolição da escravatura, mas começou, onde distante de significar uma liberdade de fato, reduziu ainda mais as relações, que os faziam permanecer aprisionados a uma sociedade de elite branca e preconceituosa (FREITAS, 2010).

Ainda que por presença imemorável da cultura de matriz europeia por força da colonização portuguesa em nosso país, a cultura tida como preponderante não conseguiu, de todo, extinguir as culturas indígena e africana. Muito pelo contrário, o colonizador europeu permitiu-se influenciar pela riqueza da pluralidade cultural de índios e negros. Contudo, o padrão de organização estabelecido pelos portugueses fez-se também presente no campo da educação e da cultura (FERNANDES, 2005).

Frequentemente, se observa o estudo da cultura, mesmo sendo essencial em uma sociedade, como algo levado em último lugar em relevância. Este modo de pensamento é visto na história tradicional que é guiada por um pressuposto teórico que orienta os pontos de vista para o enfoque socioeconômico (OLIVEIRA et al., 2012). A temática Educação para as Relações Raciais, segundo esses autores, realmente ganha escopo depois da promulgação da Lei 10.639/03 (BRASIL, 2003), que estabeleceu a obrigatoriedade da temática "História e Cultura afrobrasileira" nos currículos dos estabelecimentos de ensino de todo país.

Já no ano de 2004, nessa mesma direção, estabeleceram-se as “Diretrizes Curriculares Nacionais para a Educação das Relações Étnico-Raciais e para o Ensino de História e Cultura Afro-Brasileira e Africana" esclarecendo parâmetros que podem ser seguidos pelos sistemas de ensino para cumprir com o dever assumido: fazer justiça à história e cultura provenientes do continente africano, em contínuo desenvolvimento e transformação na diáspora negra (BRASIL, 2004).

Essa lei foi substituída pela Lei $11.645 / 2008$, que trouxe alguns aspectos que estavam presentes na antiga lei e acrescentou outros. Essa lei traz o seguinte:

"Art. 26-A. Nos estabelecimentos de ensino fundamental e de ensino médio, públicos e privados, torna-se obrigatório o estudo da história e cultura afro-brasileira e indígena.

§ 10 O conteúdo programático a que se refere este artigo incluirá diversos aspectos da história e da cultura que caracterizam a formação da população brasileira, a partir desses dois grupos étnicos, tais como o estudo da história da África e dos africanos, a luta dos negros e dos povos indígenas no Brasil, a cultura negra e indígena brasileira e o negro e o índio na formação da sociedade nacional, resgatando as suas 
contribuições nas áreas social, econômica e política, pertinentes à história do Brasil. (BRASIL, 2008).

Para Rocha (2013) trazer para sala de aula a História da África e do Brasil africano é fazer cumprir nossos objetivos como educadores: refletir sobre a discriminação racial e sexual, valorizar a diversidade étnica, gerar debate, estimular valores e comportamentos de respeito, de solidariedade, de tolerância. Além disso, mais especificamente, é a oportunidade de levantar a bandeira de combate ao racismo e às discriminações que atingem particularmente a população negra, afro-brasileira e afrodescendente. Trazer para a sala de aula esse tema é dar a oportunidade a nossos alunos de desvendarem sua própria cultura, reconhecendo no outro uma parte de si mesmos.

Para Verrangia (2009) o ensino de Ciências, assim como todos os componentes curriculares, tem papel importante na promoção de relações sociais éticas entre os estudantes. Porém, infelizmente a diversidade étnico-racial ainda não é considerada uma questão central na formação de professores dessa área, tanto inicial quanto continuada.

Portanto, os Parâmetros Curriculares Nacionais (PCNs) foram construídos, em 1997, visando de uma forma respeitar diversidades regionais, culturais e políticas existentes no país e, de outra, considerar a precisão de elaborar referências nacionais comuns ao processo educativo em todas as regiões brasileiras, permitindo criar condições nas escolas, que concedam aos nossos jovens ter acesso ao conjunto de conhecimentos socialmente elaborados e reconhecidos como necessários à prática da cidadania (BRASIL, 1997 e 1999).

No ensino de ciências, podemos utilizar dos temas transversais, um importante suporte para o processo de ensino-aprendizagem. Os temas transversais dizem respeito a conteúdos de caráter social, que devem ser inseridos no currículo do Ensino Fundamental, não como uma área de conhecimento específico, mas como conteúdo a ser tratado pelas diversas áreas do conhecimento. E de acordo com as orientações previstas nos Parâmetros Curriculares Nacionais de 1997, que determinam a inclusão da pauta dos temas transversais no Ensino Fundamental (MARTINS, 2005).

Diante disso, há uma grande importância na formação inicial de professores onde tais perspectivas centram-se na necessidade de melhor preparar tais profissionais para trabalhar juntamente com a escola para que esta seja um espaço que promova a reflexão sobre a diversidade cultural e social brasileira visando reconhecê-las e valorizá-las através de suas práticas pedagógicas.

Neste contexto, esta pesquisa teve como objetivo principal investigar se os alunos da disciplina Metodologia do Ensino em Ecologia da Universidade Federal de Lavras (UFLA), conseguiram estabelecer um diálogo entre a cultura afro-brasileira e o ensino em ecologia e como o fizeram. O estudo foi realizado sob uma perspectiva qualitativa, utilizando o procedimento de análise de conteúdo. 


\section{METODOLOGIA}

\subsection{A Disciplina de Metodologia do Ensino em Ecologia}

A disciplina de Metodologia do Ensino em Ecologia, outrora obrigatória, hoje eletiva, é ofertada no curso de licenciatura em Ciências Biológicas da UFLA. Esta tem por objetivo fazer reflexões sobre o ensino de ciências, em específico da disciplina, em ecologia, e discutir o processo de ensino-aprendizagem, afim de que os alunos da disciplina reflitam sobre sua própria prática e pensem em um ensino em que os alunos participem de forma ativa na elaboração dos conceitos, além de promover questionamentos e discussões acerca de temas que envolvem a sociedade e que se relacionam com o conteúdo biológico, afim de formar cidadãos críticos e autônomos.

Os alunos construíram um plano de aula com o conceito de Ecologia que cada um escolheu em diálogo com a cultura afro-brasileira. As atividades desenvolvidas pelos alunos se constituíram na construção de sete planos de aula, referentes à disciplina de Metodologia do Ensino em Ecologia. A proposta feita pelos alunos foi apresentada inicialmente aos bolsistas integrantes do Programa Institucional de Bolsas de Iniciação à Docência (PIBID) de Biologia da Universidade Federal de Lavras (UFLA) no IV Simpósio de Prática de Ensino de Ciências e Biologia. Os bolsistas avaliaram a prática proposta, contribuindo para a sua construção. As aulas foram realizadas para os próprios alunos da disciplina juntamente com o docente responsável. Após cada uma das aulas realizadas, os participantes as avaliaram de forma escrita, colocando os pontos positivos e os pontos a serem melhorados de cada uma, e esses serão analisados no presente trabalho.

Neste trabalho, as estratégias pedagógicas serão apresentadas, as discussões advindas das apresentações das aulas serão descritas e analisadas a fim de identificar elementos considerados importantes da cultura afro-brasileira juntamente com a disciplina Metodologia do Ensino em Ecologia.

As aulas desenvolvidas pelos alunos na disciplina estão descritas no quadro abaixo (Quadro A).

Quadro A: Aulas desenvolvidas pelos licenciandos.

\begin{tabular}{|c|c|c|}
\hline Tema & Estratégia pedagógica & Elemento cultural \\
\hline Impactos ambientais & Teatro & Religião \\
\hline Biomas & Imagens & Culinária \\
\hline Conceito de comunidades & Música & Religião \\
\hline $\begin{array}{c}\text { Teoria do forrageamento } \\
\text { ótimo }\end{array}$ & Dinâmica & Conto popular \\
\hline $\begin{array}{c}\text { Interações ecológicas } \\
\text { Conceitos de ecossistema }\end{array}$ & Conto e jogo & História do quilombo \\
\hline Populações & Fotografia & Quilombo \\
\hline
\end{tabular}




\subsection{Procedimentos de análise}

Para análise das avaliações transcritas das aulas dos participantes, utilizou-se o método de pesquisa qualitativa. Essa metodologia é aplicada quando o estudo apresenta um caráter descritivo e tem por objetivo compreender a totalidade e a complexidade do fenômeno. Segundo Godoy (1995), o pesquisador nestes casos tem como ponto central o processo e não simplesmente o produto, tentando compreender os fenômenos estudados da perspectiva dos participantes.

Dentro da perspectiva qualitativa, utilizou-se a análise de conteúdo (MINAYO, 2000) para analisar os elementos encontrados nas avaliações das aulas. A autora assegura que a mesma vem sendo um método muito empregado na análise de dados de pesquisas qualitativas.

Durante a etapa de análise, a princípio, foram analisadas todas as avaliações transcritas dos participantes da disciplina com intuito de identificar e discutir as categorias encontradas nelas. Assim, os transcritos foram agrupados em categorias tendo como base sua expressividade quantitativa, ou seja, os elementos que foram mais recorrentes e que são importantes no processo de formação inicial de professores sob uma perspectiva crítica da educação e também que expressassem o valor cultural, sendo de grande relevância o destaque da cultura afrobrasileira neste trabalho.

Em seguida, buscou-se encontrar a frequência em que cada categoria aparece nas falas dos participantes a fim de conhecer o que estes consideram mais relevante na formação de professores juntamente com elementos da cultura afro-brasileira.

\section{RESULTADOS E DISCUSSÃO}

A partir da análise das falas dos participantes, foram identificadas cinco categorias: estratégias pedagógicas, cultura afro-brasileira, contextualização, mediação do professor e diálogo entre Biologia e cultura afro-brasileira. Estas categorias estão dispostas e descritas no quadro B. Além disso, estão dispostas as frequências de cada uma dessas categorias, definidas da contabilização de cada uma delas. 
Quadro B: Categorias e frequências produzidas a partir da análise de conteúdo das avaliações.

\begin{tabular}{|c|l|c|}
\hline Categorias & \multicolumn{1}{|c|}{ Descrição das categorias } & Frequência \\
\hline Estratégias pedagógicas & $\begin{array}{l}\text { Nesta categoria se encaixam expressões em } \\
\text { que manifestações culturais podem ser } \\
\text { trabalhadas como estratégias para o ensino. }\end{array}$ & 9 \\
\hline Cultura Afro-brasileira & $\begin{array}{l}\text { Aqui é abordada a importância da valorização } \\
\text { da cultura afro-brasileira e da sua presença nos } \\
\text { estabelecimentos de ensino. }\end{array}$ & 12 \\
\hline Contextualização & $\begin{array}{l}\text { Nesta categoria encaixam-se as ideias que } \\
\text { expressam a relação entre cultura e ensino por } \\
\text { meio da contextualização dos conteúdos. }\end{array}$ & 4 \\
\hline Mediação do professor & $\begin{array}{l}\text { Esta categoria traz elementos valorizando o } \\
\text { papel do professor enquanto mediador do } \\
\text { conhecimento. }\end{array}$ & 6 \\
\hline Diálogo entre Biologia e \\
cultura Afro-brasileira & $\begin{array}{l}\text { Esta categoria traz a relação do conceito } \\
\text { Biológico com os elementos da cultura afro- } \\
\text { brasileira. }\end{array}$ & 10 \\
\hline
\end{tabular}

Após a categorização dos transcritos e da identificação das frequências, foram selecionadas algumas falas dos participantes para exemplificar e discutir as categorias.

A primeira categoria a ser discutida perpassa pela maioria das atividades desenvolvidas, pois se trata das estratégias pedagógicas, sendo a terceira maior frequência da tabela apresentada. Alguns licenciandos trazem a ideia que as estratégias pedagógicas ou recursos utilizados, pelos mesmos como sendo importantes no processo de ensino-aprendizagem como é evidenciado pelas falas a seguir:

"...O teatro, eu acho um recurso muito interessante, porque é onde todos os alunos participam e quando você vivencia, fica mais fácil de aprender. Acho que o tempo foi corrido, por isso sugiro uma maior mediação após a apresentação do teatro. Mas o restante foi muito bem elaborado e preparado".

"A prática foi bastante interessante. Trazer a música como um recurso para construir o conceito de comunidades proporcionou que os estudantes da disciplina através da interpretação das letras, dos instrumentos usados e do contexto da música construíssem o conceito proposto".

“...A parte da apresentação do teatro foi a mais envolvente e ajudou muito a fortalecer o texto que criamos". 
"A aula foi bastante interessante, principalmente pelo recurso que você trouxe a fotografia. Esse recurso possibilitou que os alunos da disciplina conhecessem um quilombo e observassem suas características e através disso o conceito de população".

“...O conto pra trazer a abordagem da cultura afro foi uma ótima ideia, pois funciona tanto para ensino médio, quanto para ensino fundamental”.

Na categoria "Estratégias pedagógicas", estão agrupadas as falas relacionadas à importância do uso de recursos pedagógicos não expositivos. Na grande maioria das escolas brasileiras ainda se faz presente o método tradicional de ensino, que conta com a exposição simplista dos conteúdos (LIBÂNEO, 1990). Frequentemente, ao trabalhar os conteúdos em sala de aula, os professores deparam-se com frágeis instrumentos de trabalho o que pode gerar dependência ao uso do livro didático, assim se limitando ao método tradicional de ensino. Nesse sentido, as estratégias pedagógicas surgem como auxílio na construção do conhecimento dos alunos sem a utilização do método expositivo. Levando em conta assim, o contexto histórico e social do aluno, contribuindo na formação de um sujeito crítico e autônomo (RICARDO, 2013).

As estratégias de ensino são ações didáticas pedagógicas intencionais, empregadas com o intuito de se conseguir melhores efeitos no método de ensino-aprendizado, e envolvem as relações professor-aluno, aluno-aluno e aluno-consigo mesmo, fundamentais para o desenvolvimento intelectual para aprender e ensinar (OKANE E TAKAHASHI, 2004). As autoras ainda argumentam que, as interações entre o professor e aluno em sala de aula são cheias de valores e significados, de subjetividades próprias de cada olhar, de propósitos, assegurando e reproduzindo no processo ensino-aprendizado um olhar especial às estratégias de ensino. $E$ ainda que para optar e aplicar estratégias, o docente além de estudar e planejar fundamentado na teoria científica, precisa juntar criatividade e adaptações em suas ações didáticopedagógicas.

Na segunda categoria "Cultura afro-brasileira", os transcritos que fazem referência a essa merecem destaque, visto que apresentaram a maior frequência de falas, mostrando como os participantes interpretaram a presença desta e de seus elementos nas aulas regidas pelos mesmos, como são evidenciadas abaixo:

"Eu gostei bastante da atividade. Pela história trazida, eu achei que conseguiu trabalhar os conteúdos e falar sobre a cultura afro-brasileira".

"Foi uma temática bem legal, as fotos foram bem bonitas e caracterizaram bem um quilombo..."

“...Interessante conhecer as entidades e o papel delas na religião umbandista. Esclarece muito a visão mística no sentido negativo que é falado no senso comum, onde podemos perceber 
que é apenas uma forma religiosa de um povo que tem origem diferente da que a visão Européia nos apresenta. Fiquei curioso para conhecer as outras características dessa religião também".

"O conto pra trazer a abordagem da cultura afro foi uma ótima ideia, pois funciona tanto para ensino médio, quanto para ensino fundamental”.

A partir de 2003 com as Leis 10.639 e em 2008 a Lei 11.645, tornou-se obrigatória nas escolas de educação básica a discussão afro-brasileira e indígena nas disciplinas de história, literatura e artes, sendo no caso da história, incluída como pontos transversais nas discussões historiográficas nas salas de aula (BRASIL, 2008). Complementar às leis que regem as diretrizes e bases da educação nacional, os Parâmetros Curriculares Nacionais (PCNs) aparecem como documentos apresentados como meta educacional, concedendo instruções às escolas em relação as suas ações. Os temas transversais trazidos por esses documentos, indispensáveis para serem discutidos nas escolas, não eventualmente ou em uma disciplina em particular, mas sim, como temáticas interdisciplinares, devem ser constantemente discutidas, seja em trabalhos, debates, informações ou sempre que algum ponto relativo aos temas for abordado em âmbito escolar (SOUZA et al., 2008). Como um dos temas transversais dos PCNs, a pluralidade cultural precisa vir a ser mais trabalhada nas escolas, onde seu caráter interdisciplinar possibilita seu tratamento dentre inúmeros campos do conhecimento, onde estes concederão diversos subsídios à temática.

Assim, segundo Souza et al. (2008), a função da escola como geradora de uma consciência pautada no conhecimento da diversidade que abrange a sociedade é definitivo, sendo que, através da presença no estabelecimento de ensino, os alunos, docentes e funcionários estão sujeitos a convívio com a diferença, tendo em mente, e compreendendo que em local público, haverá sempre o contato entre diversas raízes étnico-raciais, estilos de vida, onde o respeito mútuo deve fazer parte desse convívio. Ao levantar em sala de aula a temática da pluralidade cultural, notadamente, o professor oferece aos alunos a possibilidade de conhecer suas origens como brasileiros e como integrantes de grupos culturais específicos, valorizando sua própria cultura e apreendendo seu próprio valor, aumentando sua autoestima, e favorecendo também a valorização da cultura do outro enquanto fazem parte de diferenciados grupos sociais.

Já na terceira categoria "Contextualização", encontram-se os transcritos que trazem a importância da contextualização no processo de ensino-aprendizagem, como é evidenciado nas falas abaixo:

"Achei interessante a forma de trabalhar a antropização, pois com a história os alunos criam a continuação com aspectos que ele conhecem do dia-a-dia. O conceito foi bem compreendido". 
"A aula foi bastante interativa e permitiu a construção do conceito "população". O contexto trazido pelas fotos facilitou sua compreensão e enriqueceu a visão sobre aspectos da cultura afro-brasileira, no sentido das comunidades biológicas..."

"A aula ficou muito boa, gostei muito de como os dois temas dialogaram perfeitamente: a cultura afro-brasileira com a antropização..."

"Sua aula foi muito bacana e eu gostei da ligação que você fez da comunidade quilombola (representando a cultura afro-brasileira) com os conceitos de população como natalidade, migração definindo então o conceito de população..."

Na maior parte de nossas escolas, o ensino de ciências vem sendo praticado de modo dogmático e de maneira descontextualizada da sociedade. Os alunos não são capazes de reconhecer a relação entre o que é estudado em ciência e o seu dia a dia e, dessa forma, entendem que o estudo de ciências é resumido a guardar nomes difíceis, classificar fenômenos e a resolver problemas através de algoritmos.

Segundo Santos (2007), na maior parte de nossas escolas, os alunos não são capazes de reconhecer a relação entre o que é estudado em Ciências e o seu dia a dia e, dessa forma, entendem que o estudo de Ciências é resumido a guardar nomes difíceis, classificar fenômenos e a resolver problemas através de algoritmos. Isto posto, o autor traz que a contextualização no currículo pode ser dar através da discussão de situações reais que permeiam o cotidiano dos alunos e da escola, de modo que proporcione um debate de conceitos científicos, transpassando por eles questões sociais, ambientais, econômicas, políticas, culturais, éticas, dentre outras que se fazem presentes na nossa sociedade.

Portanto, os professores precisam levar temas que fazem parte do dia a dia dos alunos para debater conceitos ecológicos, ampliando a motivação desses pelas ciências e biologia (DEMO, 2004). Desta forma, o professor mostrará que a Biologia pode atender às necessidades humanas, trazendo questões relacionadas à ciência, à tecnologia e à sociedade.

Na penúltima categoria "Mediação do professor", traz elementos valorizando o papel do professor enquanto mediador do conhecimento como é mostrado nas falas abaixo:

\footnotetext{
"Eu achei a prática muito interessante por você perguntou inicialmente quem já conhecia a Galinha-da-angola e como ela é, seu comportamento. Assim como perguntou de outros animais que interagem com ela. Isso é válido pois valoriza o conhecimento de cada aluno."

“...O fato de trazer uma história pré-pronta e pedir para os alunos completarem foi uma ótima maneira para prender e instigar a turma a participar da aula”.

"A aula foi bastante interessante e dinâmica. Você conseguiu que os alunos participassem da prática de modo que através dessa participação, na qual cada aluno representava um orixá,
} 
você juntamente com eles construiu o conceito de forrageamento ótimo. É uma prática simples que pode ser aplicada com facilidade dentro das salas de aula."

A escola, segundo Maders (2012), é um espaço onde devem ser debatidas, de maneira mediada pelos professores, as diferentes etnias e culturas brasileiras com o objetivo de quebrar preconceitos. Logo, a escola deve ser um espaço que promova a reflexão sobre a diversidade cultural e social brasileira visando reconhecê-las e valorizá-las através de suas práticas pedagógicas.

Compreendendo que o ser humano é uma construção social e seguindo a concepção de Vygotsky, o professor enquanto mediador do conhecimento e a escola são essenciais para a formação deste sujeito social. Na função de mediador, o professor tem o papel de produzir a interação entre o aluno e o objeto de conhecimento. Ele ainda deve instigar o aluno nesse método por meio de, problematizações, questionamentos e atividades que levem o aluno a refletir (RIBEIRO, 2007). Nessa perspectiva, Bulgraen (2009) acrescenta que o professor deve se colocar como uma ligação entre o aluno e o conhecimento, a fim de que o primeiro não mais receba as informações de forma passiva, e sim aprenda a pensar e questionar.

Na última categoria apresentada "Diálogo entre Biologia e cultura afro-brasileira" apresentou a segunda maior frequência dos transcritos, e evidencia uma discussão sobre os conceitos biológicos e elementos culturais afro-brasileiros presentes no ensino. Sendo assim, as falas apresentadas abaixo mostram como a disciplina de Metodologia do Ensino em Ecologia trouxe os conceitos desta, juntamente com esses elementos da cultura em questão:

"Esse recurso possibilitou que os alunos da disciplina conhecessem um quilombo e observassem suas características e através disso o conceito de população".

"Gostei muito da ideia da aula. Você além de trazer um elemento cultural muito bacana que foi os quilombolas e uma parte da sua história de antes e como é hoje, o vídeo deu para tratar aspectos ligados ao ecossistema em que deu para ser bem compreendido..."

“Pela história trazida, eu achei que conseguiu trabalhar os conteúdos e falar sobre a cultura afro-brasileira”.

"A aula ficou muito boa, gostei muito de como os dois temas dialogaram perfeitamente: a cultura afro-brasileira com a antropização".

Para (KOEPPE et. al., 2014) inserir as temáticas culturais como a indígena e afro-brasileiras no currículo de ciências, corrobora para a importância do processo educacional no 
desenvolvimento de novos conceitos éticos, e comportamentais em relação a esses grupos, promovendo então a problematização das representações escolares equivocadas consolidadas ao longo dos anos.

Segundo Oliveira (2012) ao se falar sobre conteúdos e metodologias que valorizem as relações étnico-raciais, possibilita e incentiva o trabalho conjunto entre duas ou mais áreas diferentes, na procura de um trabalho interdisciplinar. Ainda, o conteúdo sobre as relações étnico-raciais requer uma atenção especial, em razão do modo em que foi formada a sociedade brasileira e as maneiras de manifestação do racismo.

A escola desempenha um papel essencial, visto que é diretamente ela que trabalha na formação intelectual dos cidadãos brasileiros sendo, desse modo considerada o principal local para articular o debate étnico e causar uma mudança social que será refletida na cultura do povo (OLIVEIRA et al., 2008).

\section{CONSIDERAÇÕES FINAIS}

Diante da análise das falas dos discentes da Disciplina Metodologia do Ensino em Ecologia e das cinco categorias identificadas, conclui-se que essa juntamente com o tema transversal pluralidade cultural proporciona aos participantes um maior conhecimento sobre a cultura afrobrasileira corroborando para a formação inicial de professores. Pois, frequentemente, se observa o estudo da cultura como algo levado em último lugar em importância, sendo essencial em uma sociedade. A disciplina fez com que os licenciandos trabalhassem o tema transversal e a cultura afro-brasileira em questão preparando-os para atuar criticamente na escola, pois é nela que se trabalha a formação intelectual dos cidadãos sendo, desse modo considerada o principal local para articular um debate étnico e causar uma mudança social. É também de grande relevância para que se desenvolva a criticidade de professores ainda no processo de formação, para que estes superem as dificuldades postas na escola, com temas cruciais vividos por alunos como discriminação racial, sexual, religiosa entre outras.

Além disso, trabalhar temas como a cultura afro-brasileira é importante não apenas para a construção e valorização de uma identidade cultural que ressalte a história e cultura dos povos que contribuíram para a formação da população brasileira, mas também para não deixar perder saberes que foram passados ao longe de gerações como o conhecimento de plantas medicinais, suas crenças, seus mitos, seus valores, suas lutas, sua história.

Por fim, ensinar Ciências e Biologia representa um desafio para os docentes, uma vez que seu conteúdo é de difícil entendimento por parte dos alunos. Pensar em meios de realizar a interrelação entre sala de aula e cotidiano é pensar em novas formas didáticas e metodológicas. Assim, a disciplina de Metodologia do Ensino em Ecologia leva ao desenvolvimento de metodologias que utilizam recursos pedagógicos não convencionais, que atuam como facilitador no processo de ensino-aprendizagem, para o ensino do conteúdo ecológico e também sendo essas de grande importância na construção do conhecimento por parte do aluno. Ela também faz com que os discentes participantes da atividade elaborem aulas mais dinâmicas e acima de tudo não expositivas, que permitam a criatividade, aprendizagem e o desenvolvimento de 
metodologias que utilizem estratégias pedagógicas não tradicionais, sendo essencial para um ensino mais eficiente do conteúdo obrigatório a ser trabalhado em sala de aula.

\section{REFERÊNCIAS}

BRASIL. MEC. LDB - Lei no 9394/96, de 20 de Dezembro de 1996. Estabelece as diretrizes e Bases da Educação Nacional. Brasília: MEC, 1996. de dez. 2015.

Lei no. 10.639, de 9 de janeiro de 2003. Disponível em: <http://www2.planalto.gov.br/>. Acesso em: 18

. Lei no 11.645 de 10 de março de 2008. Disponível em: <http://www.planalto.gov.br/ccivil_03/_ato2007 2010/2008/lei/l11645.htm>. Acesso em: 18 de dez. 2015.

Ministério da Educação. Diretrizes Curriculares Nacionais para a Educação das Relações Étnico-Raciais e para o Ensino de História e Cultura Afro-Brasileira e Africana. Brasília-DF, Outubro, 2004.

Ministério da Educação. Secretaria de Educação Fundamental. Parâmetros curriculares nacionais: pluralidade cultural, orientação sexual. Brasília, DF: MEC/SEF, 1997.

BULGRAEN, Vanessa Cristina; O Papel do Professor e Sua Mediação nos Processos de Elaboração do Conhecimento. Revista Conteúdo, Capivari, v.1, n.4, ago./dez. 2010.

DEMO, Pedro; Educação e qualidade. Campinas: Papirus, 1996. v. 1. 160 p.

FERNANDES, José Ricardo Oriá; Ensino de história e diversidade cultural: desafios e possibilidades; Cadernos Cedes, Campinas, vol. 25, n. 67, p. 378-388; Campinas-SP, 2005

GODOY, Arlida Schmidt; Introdução à pesquisa qualitativa e suas possibilidades. Em: Revista de Administração de Empresas, São Paulo, v. 35, p. 57-63, 1995.

KOEPPE, Cleise Helen Botelho; BORGES, Regina Rabello.; LAHM, Regis. O Ensino de Ciências como ferramenta pedagógica de reconstrução das representações escolares sobre os povos indígenas. Revista Ensaio, v.16, n. 01, p. 115-130; Belo Horizonte- MG, janeiro-abril/2014.

LIBÂNEO, J. C. Democratização da Escola Pública: a pedagogia crítico-social dos conteúdos. São Paulo: Loyola, 1990. 160p.

MADERS, Sandra; Educação Escolar Indígena e Inclusão - por uma pedagogia do cuidado e da escuta.. In: IX Seminário de Pesquisa em Educação da Região Sul - ANPED SUL, 2012, Caxias do Sul. Anais: IX Seminário de Pesquisa em Educação da Região Sul - ANPED SUL, 2012. p. 1-15.

MARTINS, Luzenice Macedo; Temas transversais para professores de Ciências. Revista comunicação \& educação, São Paulo v.5, n.2, p 219-224, maio/ago 2005.

MINAYO, Maria Cecília de Souza; O desafio do conhecimento: pesquisa qualitativa em saúde. 7. ed. São Paulo: Hucitec, 2000. 269 p.

NASCIMENTO, José Antônio; Moraes do. História e cultura indígena na sala de aula. Revista Latino-Americana de História, v. 2, n. 6, p.150, 2013.

OKANE, Eliana Suemi Handa; TAKHASHI, Regina Toshie; O estudo dirigido como estratégia de ensino na educação profissional em enfermagem. Anais VIII Seminário Nacional de Diretrizes para a Educação em Enfermagem, Vitória - ES, 2004. 
OLIVEIRA, Liélia Barbosa; OLIVEIRA Thomas Bruno; ARAGÃO Patrícia Cristina de Araújo; Transversalidade cultural: notas sobre a prática de ensino e a temática afro-brasileira e indígena nas salas de aula. UEPB. 2008.

OLIVEIRA, Leila Maria de; A Interdisciplinaridade Transversalidade na abordagem da educação para as Relações Étnico-Raciais. Curso Educação, Relações Raciais e Direitos Humanos. São Paulo, 2012.

RIBEIRO, Elizabeth da Cruz; A Prática Pedagógica do Professor Mediador na Perspectiva de Vygostky. 2007. 42 f. Monografia (Especialização) - Curso de Psicopedagogia, Universidade Candido Mendes, Rio de Janeiro, 2007.

ROCHA, Aristeu Castilhos da; Desafios para o Ensino de História e Cultura Afro-brasileira e Indígena. In: XVI Jornada Nacional de Educação, 2012, Santa Maria, RS. Anais da XVI Jornada Nacional de Educação, 2012.

SANTOS, Wildson Luiz Pereira; Contextualização no ensino de ciências por meio de temas CTS em uma perspectiva crítica. Ciência \& Ensino (UNICAMP. Impresso), v. 1, p. 1-12, 2007.

SOUZA, Andrêza Gomes de; GOUVEIA, Camilla. Ferreira; LOPES, Michelly de Lourdes; A Ética e a Pluralidade Cultural Contribuindo na Formação de Cidadãos. In: IV SIMPÓSIO INTERNACIONAL: O Estado e as Políticas Educacionais no Tempo Presente. Uberlândia, 2008.

VERRANGIA, Douglas Corrêa da Silva; Conhecimentos tradicionais de matriz africana e afro-brasileira no ensino de Ciências: um grande desafio. Revista África e Africanidades v. 8, p. 14, 2010. 